\title{
La comunicación online del Cambio Climático en España
}

\section{The online Climate Change Communication in Spain}

\author{
Gemma Teso Alonso. Universidad Complutense de Madrid. España \\ gtesoalonso@gmail.com \\ $[\underline{\mathrm{CV}}] \odot \mathrm{G}$ \\ Carlos Lozano Ascencio. Universidad Rey Juan Carlos. España \\ carlozanoas@gmail.com \\ $[\mathrm{CV}] \mathrm{C}$
}

Este artículo ha sido elaborado con los datos del Observatorio de la Comunicación del cambio climático en España puesto en marcha por el grupo de investigación MDCS de la Universidad Complutense y la Fundación Ecología y Desarrollo (ECODES), con la financiación del Ministerio para la Transición Ecológica (MITECO) mediante las ayudas a proyectos de investigación 2019 y 2020.

Cómo citar este artículo / Referencia normalizada

Teso-Alonso, G., y Lozano Ascencio, C. (2022). La comunicación online del Cambio Climático en España. Revista Latina de Comunicación Social, 80, 65-87. https://www.doi.org/10.4185/RLCS-2022-1531

\begin{abstract}
RESUMEN
Introducción: La evolución de la cobertura informativa del cambio climático durante los últimos veinte años coincide con el surgimiento, expansión y consolidación de los medios digitales en España. El objetivo de este estudio es explorar la comunicación online del cambio climático en España identificando las principales fuentes de información y las características de la comunicación que practican. Metodología: Desde un enfoque cuantitativo, se analizan todos los registros obtenidos durante la primera sonda online realizada por El Observatorio de la Comunicación del Cambio Climático durante la primavera-verano de 2019 (fuente de datos: Kantar Media). Se aplica un protocolo de análisis de contenido para identificar las fuentes de información sobre CC en Internet y el contenido temático. Resultados: Se encuentra una tendencia a similar a la de los medios convencionales en cuanto a la estacionalidad, horarios y cobertura. Se identifican las principales fuentes de información generalistas y especializadas estableciendo un ranking con las treinta más relevantes y se analiza el "sentimiento" asociado a los discursos. Discusión: La prensa generalista es la líder indiscutible en el entorno online ante el escaso volumen de publicaciones sobre cambio climático de la radio y la televisión en Internet. Junto con la prensa, asociaciones sectoriales, empresas e instituciones municipales o autonómicas son las principales fuentes de información especializada. La agencia de noticias Europa Press ocupa el primer lugar por volumen de información publicada en el ranking de las treinta principales fuentes de información online. Conclusiones: Encontramos gran transversalidad del cambio climático como referencia informativa, que es comunicado por sectores especializados como el económico y financiero y por medios diversos concernidos por la transformación ecológica en el contexto social de emergencia climática de 2019.
\end{abstract}


PALABRAS CLAVE: comunicación online; cambio climático; fuentes informativas; cibermedios, emergencia climática; sectores; sentimiento.

\begin{abstract}
Introduction: The evolution of news coverage of climate change during the last twenty years coincides with the emergence, expansion and consolidation of digital media in Spain. The objective of this study is to explore online communication on climate change in Spain, identifying the main sources of information and the characteristics of the communication they practice. Methodology: From a quantitative approach, all the records obtained during the first online probe carried out by the Observatory for Climate Change Communication are analyzed (data from Kantar Media). Results: There is a trend similar to conventional media in terms of seasonality, schedules and coverage. The main general and specialized sources of information are identified, establishing a ranking with the thirty most relevant and the "sentiment" associated with the speeches is analyzed. A content analysis protocol is applied to identify the sources of information about CC on the Internet and the thematic content. Discussion: The general press is the undisputed leader in the online environment due to the low volume of publications on climate change on radio and television on the Internet. Sectorial associations, companies and municipal or regional institutions are the main sources of specialized information together with the press. The Europa Press news agency ranks first in terms of volume of information published in the ranking of the thirty main sources of online information. Conclusions: We find great transversality of climate change as an informative reference, which is communicated by specialized sectors such as the economic and financial sector and by various media concerned by the ecological transformation in the social context of the climate emergency of 2019.
\end{abstract}

KEYWORDS: online communication; climate change; information sources; cybermedia; climate emergency; sectors; sentiment.

\title{
CONTENIDO
}

1. Introducción. 2. Objetivos. 3. Metodología. 4. Resultados. 5. Discusión. 6. Conclusiones. 7. Bibliografía. 8. Currículum Vitae.

\section{Introducción}

\subsection{La cobertura informativa del cambio climático en España en los últimos veinte años}

En este apartado se va a realizar una concisa revisión de la evolución de la cobertura informativa del cambio climático durante los últimos veinte años, periodo de tiempo que coincide con el surgimiento, expansión y consolidación de los medios digitales en España. A continuación, revisaremos las conexiones entre ambos fenómenos a través de la respuesta de la audiencia, es decir, se expondrá cómo la ciudadanía emplea los medios, convencionales y online, para acceder a la información sobre el cambio climático. Este recorrido nos servirá para contextualizar el objeto de estudio que no es otro que la comunicación online del cambio climático en España.

La comunicación del cambio climático durante los últimos veinte años en los medios convencionales puede ser calificada, en términos generales, como una larga y prolongada irrelevancia mediática hasta la llegada del año 2018. Exceptuando picos de mayor cobertura, el cambio climático ha sido tradicionalmente objeto de escaso seguimiento por parte de los medios españoles (Parratt, 2009; Fernández y Jiménez, 2020; Francescutti et. al, 2013; Lopera, 2013; Gaitán \& Piñuel, 2013; Lozano, 2013; Mancinas, 2013; León \& Erviti, 2015; Heras, Meira \& Benayas, 2016; Teso, 2016). Además de la cobertura anual de las cumbres del clima como eventos políticos de escala internacional, podemos señalar algunos hitos en la cobertura mediática del cambio climático, entre los que destaca 
el año 2007. En este año coincidieron el lanzamiento internacional del documental "Una verdad incómoda" protagonizado por Al Gore con la entrega del premio Nobel de la Paz al Panel Intergubernamental de expertos sobre el Cambio Climático (IPCC) de las Naciones Unidas y al propio Al Gore.

Durante la celebración de la cumbre de Copenhague celebrada en diciembre de 2009 se produjo la detención de Juantxo López de Uralde junto a otra activista de Greenpeace, quienes lograron infiltrarse en la cena de gala para altos mandatarios con el objetivo de visibilizar su petición de compromisos concretos y acción climática. El referido acontecimiento gozó de amplia cobertura internacional, si bien la llegada inmediata de la crisis económica de 2008 silenció el cambio climático en los medios durante más de cuatro años. El quinto informe del IPCC, que se publicó en los años 2013 y 2014, alentaba sobre la gravedad y urgencia de acometer medidas para frenar la crisis climática, pero hubo que esperar a finales de 2015 para que el nuevo Acuerdo internacional firmado durante la celebración de la Cumbre de París (Paris Agreement) volviera a situar el cambio climático con fuerza en el escenario mediático nacional e internacional. En 2016, la Carta Encíclica Laudato Sí del Papa Francisco "Sobre el cuidado de la casa común" (2016) ${ }^{1}$ permitió visibilizar la dimensión de la ética y de la justicia en el problema del cambio climático. Los mensajes de Donald Trump llamaron la atención de los medios en 2016 y 2017 por su pertinaz negacionismo, al que se suman voces de otros mandatarios como Jair Bolsonario (Rejón, 2018).

El mensaje de alarma del informe del IPCC $+1,5^{\circ} \mathrm{C}$ publicado en 2018 fue amplificado en las calles por nuevos movimientos estudiantiles y sociales Fridays For Future y Extinction Rebellion. En 2019 se unieron a estas voces otros grupos de apoyo como Madres por el Clima o Teachers For Future, quienes alzaron su voz ante la alarma social provocada por el evidente deterioro del planeta. En septiembre del año 2019 tiene lugar la Semana de Acción Climática, segmento temporal en el que coinciden la cumbre de la Acción Climática convocada por Naciones Unidas el lunes día 23, la presentación del Informe del IPCC sobre el impacto del cambio climático en los océanos (IPCC, 2019) que tuvo lugar el miércoles 25 , y la celebración de la multitudinaria huelga mundial por el clima celebrada al unísono en 150 países el día 27 de septiembre. Esa semana, del 23 al 29 de septiembre de 2019, contribuyó a crear un ambiente propicio para la celebración de la COP 25 Madrid- Chile que tuvo lugar en Madrid, acontecimiento que se convirtió en un verdadero tsunami informativo en España a finales de 2019 (Fernández-Reyes \& Teso, 2020). La llegada de la crisis sanitaria del Covid 19 en marzo de 2020 y la declaración del estado de alarma sanitaria hicieron retroceder el protagonismo del cambio climático en los medios a niveles anteriores a 2018 (Fernández-Reyes \& Jiménez, 2020).

\subsection{La evolución de los medios digitales en su primer cuarto de siglo}

Paralela a la historia de la cobertura del cambio climático en los medios, encontramos la evolución de los medios digitales en su primer cuarto de siglo. Salaverría (2020) es partidario de emplear el término periodismo digital para referirse a la práctica del periodismo en el entorno online, aunque otros autores sean partidarios de términos "such as digital journalism (Kawamoto, 2003), cyber journalism (Díaz-Noci \& Salaverría, 2003), online journalism (Deuze, 2001; Steensen, 2011), multimedia journalism (Deuze, 2004), and a long list of similar labels (Karlsen \& Stavelin, 2014)”. (Salaverría 2020, p. 2).

\footnotetext{
${ }^{1}$ Texto íntegro de La Carta Encíclica Laudato Sí disponible en: https://www.vidanuevadigital.com/wpcontent/uploads/2015/06/Laudato-Si-ES.pdf
} 
La llegada de la crisis económica en 2008 no sólo impactó en la cobertura mediática del cambio climático, también lo hizo en las propias estructuras empresariales de los medios. El crecimiento de medios en el entorno online creció en gran medida por haber coincidido con una gran crisis económica que redujo los ingresos de los medios tradicionales obligándoles a reducir sus redacciones (Díaz, 2011). La Asociación de la Prensa de Madrid comenzó en 2008 a elaborar un listado de los nuevos medios puestos en marcha por periodistas desde el comienzo de la gran crisis económica. Ese listado es revisado y actualizado en los Informes de la Profesión Periodística ${ }^{2}$ que emite anualmente esta Asociación (años 2013, 2014 y 2015). La edición de 2019 realizó un nuevo recuento y revisión de dichos medios. El número total de medios y otros proyectos periodísticos lanzados entre 2008 y 2015 fue de 579. En octubre de 2019, de esos 579 medios, el 52,15 \% permanecían activos; el 35,9 $\%$ habían cerrado o dejado de actualizarse; el 5,51\% se actualizaban esporádicamente y el 6,39\% se habían transformado en blogs o en webs de contenido patrocinado.

Tal y como señalan Salaverría, Martínez- Costa \& Breiner (2018), la crisis económica no fue la única causante del crecimiento constante de los medios digitales. El desarrollo tecnológico aplicado a la digitalización de la información ha situado a Internet como un escenario propicio para la convergencia mediática, tanto de los nuevos medios como de los medios convencionales. Por otro lado, los dispositivos móviles han transformado las formas de consumo (López, 2015; Meso, 2006; Yunquera, 2016) potenciado la accesibilidad y la inmediatez en el consumo de información.

Salaverría, Martínez-Costa y Breiner (2018) llevaron a cabo un exhaustivo mapa de los cibermedios o medios digitales de España en 2018 mediante un análisis cuantitativo de los medios digitales. Estos autores identificaron un total de 3.431 medios. De ellos, estaba activo el 89,3\% (3.065), frente a un $10,7 \%$ (366) que no registraba actividad. De los medios digitales activos, un total de 1.077 (35,1\%) eran nativos digitales, frente a $1.559(50,9 \%)$ que eran medios no nativos. Los cibermedios activos $(1809$, el $60 \%)$ combinaban la publicación online con la presencia en dos, tres o cuatro plataformas (prensa, radio, televisión y/o aplicaciones móviles). Con respecto al contenido, una amplia mayoría de los medios se decanta por la información general $(68,5 \%)$. Los autores identificaron un auge de los medios digitales locales que abordan la información de proximidad desde un enfoque generalista. Por su parte, percibieron un descenso de los medios especializados que pasaron de un 40,3\% en 2005 a un 31,5\% en 2018. Las temáticas con más presencia entre ellos son cultura (30\%), deportes $(14,6 \%)$, publicaciones técnicas y profesionales $(10,2 \%)$, y ocio y entretenimiento $(9,8 \%)$. La economía $(7,9 \%)$ y la ciencia y tecnología $(3,8 \%)$ acumulaban porcentajes relativamente bajos del total.

\subsection{Las principales fuentes de información sobre el cambio climático para la ciudadanía en 2020}

Para concluir la contextualización del objeto de estudio que se aborda en esta investigación es necesario abordar ahora la respuesta de la ciudadanía al consumo de medios cuando la información que se publica es el cambio climático. El estudio transnacional Digital News Report 2020 (Newman, Fletcher et ál., 2020) publicado por el Instituto Reuters de la Universidad de Oxford nos informa sobre las principales fuentes de información sobre el cambio climático que emplea la ciudadanía de los países participantes en el Estudio (véase Imagen 1). El informe señala que el porcentaje de la población española que emplea las noticias televisivas con el fin de estar informado es del $71 \%$, a la vez que el $83 \%$ accede a los medios online con el mismo fin, incluyendo las redes sociales. Similares porcentajes se presentan en otros países de Europa como Gran Bretaña o Alemania. Se

${ }^{2}$ Informes disponibles en: https://www.apmadrid.es/publicaciones/informe-anual-de-la-profesion/ 
RLCS, Revista Latina de Comunicación Social, 80, 65-87

[Investigación] DOI: 10.4185/RLCS-2022-1531| ISSN 1138-5820| Año 2022

trata por tanto de un consumo multipantalla asociado a una hiper conectividad sin horarios en la mayor parte de los países de un mundo globalizado.

\section{PROPORTION THAT USED EACH AS A SOURCE OF NEWS IN THE LAST WEEK (APRIL 2020) - SELECTED COUNTRIES}

\begin{tabular}{l|c|c|c|c|c}
\hline & $\begin{array}{c}\text { Online } \\
\text { (incl. social } \\
\text { media) }\end{array}$ & TV & $\begin{array}{c}\text { Social } \\
\text { media }\end{array}$ & Radio & $\begin{array}{c}\text { Print } \\
\text { (incl. mags) }\end{array}$ \\
\hline G UK & $79 \%$ & $71 \%$ & $47 \%$ & $35 \%$ & $18 \%$ \\
\hline S Spain & $73 \%$ & $60 \%$ & $47 \%$ & $21 \%$ & $16 \%$ \\
\hline Avermany & $69 \%$ & $72 \%$ & $39 \%$ & $41 \%$ & $26 \%$ \\
\hline $\begin{array}{l}\text { Average change } \\
\text { from January }\end{array}$ & $83 \%$ & $71 \%$ & $63 \%$ & $24 \%$ & $28 \%$ \\
\hline
\end{tabular}

Q4. (Apr. 2020). Which, if any, of the following have you used in the last week as a source of news? Base: Total sample: UK = 2191, USA = 1221, Germany = 2003, Spain =1018, South Korea = 1009, Argentina $=1003$. Note. Figures adjusted to exclude non-news users for comparability.

Imagen 1. Porcentaje de población que ha accedido a los distintos soportes (Televisión, radio, prensa, redes sociales y medios online) como fuentes de información sobre cambio climático

Fuente: Reuters Institute for the Study of Journalism. University of Oxford (2020, p. 10)

Los datos globales proporcionados por los encuestados en este estudio transnacional indican que la población presta más atención a la información del cambio climático emitida por la televisión convencional que a ninguna otra fuente de información (35\%). Como segunda fuente de información más importante $(15 \%)$ se sitúan los sitios web de los principales medios de comunicación (televisión, radio y prensa on line). En tercera posición se sitúan los sitios web de medios específicos sobre temas climáticos (13\%) y en cuarto lugar las redes sociales y los blogs $(9 \%)$, seguidos de las conversaciones con amigos y familiares como fuente de información (6\%). La prensa impresa y la radio convencional son señaladas como la quinta fuente de información sobre el cambio climático para un $5 \%$ de los encuestados respectivamente, mientras que un $7 \%$ confiesa no prestar atención al cambio climático. Los jóvenes de la Generación $Z$ (18-25 años) participantes en este estudio explicaron sus motivaciones para emplear las redes sociales, mostrando su preferencia por el entorno digital porque les permite acceder a un amplio rango de fuentes además de a los principales medios tradicionales. Les interesan la información publicada con un punto de vista concreto y poder consultar fuentes digitales con diversas perspectivas.

\subsection{La comunicación del cambio climático en Internet}

Entre los estudios llevados a cabo sobre la comunicación del cambio climático en Internet destacan tres por su relación con el objeto y enfoque del estudio que aquí se presenta.

La Dra. Joanna Boehnert del Center for Science and Technology Policy Research del Cooperative Institute for Research in Environmental Sciences (CIRES) de la Universidad de Colorado llevó a cabo en 2014 un exhaustivo estudio sobre las fuentes de información sobre cambio climático en Estados Unidos, Reino Unido y Canadá, identificando a los principales actores y sus redes de 
información. El resultado es una gran infografía que sirve como mapa de la comunicación del cambio climático en los países señalados, incluyendo en su estudio tanto a los medios convencionales como a las diversas fuentes de información presentes en Internet ${ }^{3}$. En su estudio, La Dra. Joanna Boehnert llevó a cabo una categorización de las distintas fuentes de información del cambio climático, cuyo listado se relaciona a continuación:

1. government

2. intergovernmental organization

3. association

4. scientific research

5. media

6. NGO / charity

7. research institute

8. website or blog

9. contrarian organization

10. contrarian blog

11. individual

12. corporation

El segundo estudio corresponda al análisis realizador por Arcila et ál. (2015) sobre la cobertura del cambio climático llevada a cabo por los medios online de habla hispana. Este estudio se centra en el seguimiento de las cumbres de Cancún (2010) y Durban (2011), comparando la cobertura que llevan a cabo los medios convencionales y los sitios web de noticias. Los resultados concluyen que la cobertura de los medios convencionales de las cumbres de Cancún (2010) y Durban (2011) y la cobertura de los sitios web de noticias mostraron similares tendencias. En pleno contexto social de crisis económica, tanto los medios convencionales como los medios digitales redujeron la cobertura informativa de la cumbre de Durban. Esta tendencia fue sensiblemente más acusada aún en los medios digitales que en los medios convencionales (Arcila et ál., 2015).

Por último, destacamos el estudio titulado Dominant counter-frames in influential climate contrarian European think tanks de Almirón, Boykoff, et ál. (2020) que identifica los ocho think tanks negacionistas más importantes de Europa que publican en inglés, castellano, alemán y francés. La mayor parte de estos think tanks (laboratorios de ideas, en castellano) se fundaron entre 2003 y 2009, pero su actividad se incrementó en el periodo comprendido entre 2014 y 2018. Los autores analizaron 1.669 textos y detectaron que los informes del IPCC AR4 y AR5, emitidos en 2007 y en 2014 respectivamente, sirvieron como detonante para estos laboratorios negacionistas europeos. El segundo think tank más importante de Europa por su volumen de publicaciones es el instituto Juan de Mariana, con sede en Madrid. El estudio encontró 112 textos publicados entre 2001 y 2018 en su página web.

\section{Objetivos y preguntas de investigación}

El objetivo general de este estudio es explorar la comunicación online del cambio climático en España en el año 2019, en el contexto social de emergencia climática anterior a la llegada de la pandemia causada por el SarsCov-2 en marzo de 2020. Este objetivo general se concreta en los siguientes objetivos específicos:

O.1. Identificar y clasificar las principales fuentes de información sobre el CC en el entorno online no incluyendo en el estudio las redes sociales (RRSS)

\footnotetext{
${ }^{3}$ Este completo mapa se encuentra disponible en: Disponible en: http://ecolabsblog.wordpress.com
} 

O.2. Identificar a los principales medios, organizaciones e instituciones que comunican sobre el cambio climático en el entorno online y su ámbito o cobertura territorial de interés.
O.3. Identificar las principales temáticas que se comunican en relación con el cambio climático.
O.4. Formular nuevas hipótesis de trabajo para estudios posteriores.

Las preguntas de investigación que han orientado este estudio exploratorio han sido las siguientes:

P.1. Teniendo en cuenta el volumen de medios online emergentes en la última década en España, la primera pregunta es si son también los medios de comunicación (radio, televisión, prensa) los principales emisores de información sobre el cambio climático en Internet.

P.2. En segundo lugar, nos cuestionamos sobre cuáles son los agentes sociales e institucionales de España que se comportan como fuentes de información sobre cambio climático en Internet y las características generales de su comunicación.

P.3. Nos preguntamos por los temas en los que están especializadas las diversas fuentes de información online que comunican el cambio climático en el periodo analizado.

\section{Metodología}

\subsection{Técnica de análisis y muestreo}

El objetivo de este estudio es de naturaleza exploratoria y descriptiva con un enfoque cuantitativo, ya que se pretende analizar los datos obtenidos para explorar la estructura que subyace al conjunto. A continuación, se describen las características de las fuentes de información sobre el cambio climático identificadas en Internet (excluyendo las Redes Sociales). Con este propósito, se ha aplicado la técnica de análisis de contenido, que ha permitido identificar los principios generales del fenómeno que se estudia.

Al tratar de analizar un área de la comunicación del cambio climático poco estudiada hasta la fecha, se ha optado por un muestreo no probabilístico de carácter estratégico en virtud de los segmentos temporales elegidos. El Observatorio de la Comunicación Mediática del Cambio Climático en España realizó tres sondas online durante el año 2019: la primera tuvo lugar en la primavera-verano, la segunda en el verano-otoño y la tercera en otoño-invierno (que incluyó la cobertura de la COP 25 en Madrid).

Se ha elegido la primera sonda de 2019 para la exploración de la comunicación del cambio climático en el mundo online porque se busca identificar aquellas fuentes que comunican el fenómeno del cambio climático al margen del impacto del acontecer noticioso. La segunda sonda, la de veranootoño, tuvo lugar durante el mes de septiembre de 2019 e incluía la denominada Semana de Acción Climática, que fue preparada concienzudamente por los activistas en todo el mundo y a la que se sumaron numerosos eventos como la Cumbre de la Acción Climática organizada por Naciones Unidas en Nueva York o la presentación del Informe IPCC (2019) sobre el impacto del cambio climático en los océanos. La tercera sonda, la de invierno, incluyó los preparativos y la celebración de la cumbre del clima Madrid-Chile (2019). El cambio climático se convirtió entonces en un tema prioritario de la agenda política y social y recabó una inusitada cobertura informativa (FernándezReyes, \& Teso, 2019).

\subsection{Fuente de datos y objeto de estudio}

La fuente de datos del Observatorio de la Comunicación del Cambio Climático ha sido la empresa Kantar Media, contratada para realizar el seguimiento de los medios convencionales y de las fuentes online en España. Los filtros aplicados para la selección de las publicaciones han sido los términos: 
cambio climático, crisis climática, emergencia climática y calentamiento global. Quedan excluidas de este estudio las Redes Sociales.

El objeto de estudio analizado está formado por todas las informaciones sobre cambio climático publicadas en Internet durante la primera sonda del año 2019. Para esta primera sonda se registraron todas las publicaciones sobre cambio climático divulgadas en el entorno online durante treinta y cinco días consecutivos comprendidos entre el quince de junio y el veinte de julio de 2019. Se identificaron un total de 2651 informaciones que han constituido las 2651 unidades de análisis de este estudio.

Las tablas y gráficos que se presentan a continuación han sido elaborados por los autores de este texto y miembros del equipo de investigación del citado Observatorio, a partir de los datos primarios obtenidos por Kantar Media.

\subsection{Variables de análisis}

Las variables de análisis empleadas han sido establecidas en virtud de los datos que ofrece la propia fuente online en relación con su identidad y actividad. En primer lugar, se ha procedido a estudiar el tipo de datos que muestran las distintas fuentes de información que publicaron contenidos sobre cambio climático en Internet. Se ha elegido trabajar con los datos cuantitativos que permiten identificar las distintas fuentes de información y clasificarlas en base a la información aportada por la propia fuente. No se han empleado variables de análisis de corte cualitativo. Las variables de análisis aplicadas han sido las siguientes:

1. Fecha de la publicación.

2. Nombre de la fuente

3. Tipología de fuente. Se clasifican las fuentes atendiendo su actividad principal, diferenciando si se trata de medios de comunicación profesionales o si son instituciones de otra naturaleza cuya función principal no es la comunicación pública, si bien también divulgan contenidos en el entorno online (véase el caso de ONGs, empresas, sindicatos, etc.).

a. Prensa (diario, semanario, otras). Esta categoría comprende las versiones digitales de los diarios convencionales y los diarios nativos digitales.

b. Radio.

c. Televisión.

d. Agencia.

e. Revista de medios (no generan contenido propio, sino que recogen lo publicado por otros medios).

f. Medio online-híbrido (produce información en diversos soportes: audio, vídeo, texto, fotos, eventos, etcétera). Hemos denominado "híbridos" a los medios que no pueden clasificarse en una sola de las categorías anteriores.

g. Instituciones/organizaciones/asociaciones que no son medios de comunicación: Empresas; Sindicatos; Fundaciones; Centros investigación; ONG; Asociaciones sectoriales; Gobiernos (regionales, locales, nacionales).

h. Otras.

4. Cobertura territorial de interés para la fuente. Teniendo en cuenta que las publicaciones en el entorno online superan los límites territoriales de cobertura de los medios lineales, esta variable guarda relación con la idiosincrasia de la entidad que comunica y su interés en la cobertura informativa de contenidos de ámbito internacional, nacional, regional o local, dependiendo del área geográfica de la audiencia a la que se dirigen.

5. Tipo de contenido que publica la fuente:

a. Generalista 
b. Especializado.

6. Si la fuente publica contenido especializados, se indica el tema. Esta variable ofrece una categorización de gran heterogeneidad: Economía; Deportes; Sector primario (agricultura, ganadería y pesca); Defensa, Energía; Mercado y Bolsa; Cultura; etc.

\section{Resultados}

\subsection{Estacionalidad $\mathbf{y}$ horarios}

Se obtuvieron un total de 2651 informaciones en total repartidas entre los 35 días de la primera sonda de manera que se encontraron 1358 unidades de análisis en junio de 2019, el 51,2 \% de total, y 1293 unidades en julio de 2019, el 48,8 \% del total. Esto hace un promedio de 90,5 informaciones por día durante el mes de junio y 64,6 durante los días del mes de julio, lo que indica una tendencia al descenso en el volumen de la información con la llegada del verano.

Tabla 1. Días de seguimiento de la cobertura del cambio climático en medios convencionales y en Internet de la primera sonda llevada a cabo en 2019

\begin{tabular}{|c|c|c|c|}
\hline \multicolumn{5}{|c|}{ Días registrados de la $1^{\mathbf{a}}$ sonda } \\
\hline de 2019 \\
\hline Día & Junio & Julio & Total \\
\hline 1 & 0 & 63 & 63 \\
\hline 2 & 0 & 81 & 81 \\
\hline 3 & 0 & 84 & 84 \\
\hline 4 & 0 & 73 & 73 \\
\hline 5 & 0 & 108 & 108 \\
\hline 6 & 0 & 43 & 43 \\
\hline 7 & 0 & 48 & 48 \\
\hline 8 & 0 & 44 & 44 \\
\hline 9 & 0 & 104 & 104 \\
\hline 10 & 0 & 79 & 79 \\
\hline 11 & 0 & 83 & 83 \\
\hline 12 & 0 & 53 & 53 \\
\hline 13 & 0 & 34 & 34 \\
\hline 14 & 0 & 72 & 72 \\
\hline 15 & 0 & 106 & 106 \\
\hline 16 & 28 & 59 & 87 \\
\hline 17 & 106 & 61 & 167 \\
\hline 18 & 166 & 34 & 200 \\
\hline 19 & 170 & 59 & 229 \\
\hline 20 & 65 & 5 & 70 \\
\hline 21 & 70 & 0 & 70 \\
\hline 22 & 21 & 0 & 21 \\
\hline 23 & 16 & 0 & 16 \\
\hline 24 & 119 & 0 & 119 \\
\hline 25 & 116 & 0 & 116 \\
\hline 26 & 154 & 0 & 154 \\
\hline 27 & 122 & 0 & 122 \\
\hline 28 & 92 & 0 & 92 \\
\hline 29 & 86 & 0 & 86 \\
\hline 30 & 27 & 0 & 27 \\
\hline & 1358 & 1293 & 2651 \\
\hline & & & \\
\hline
\end{tabular}

Fuente: elaboración propia 
La mayor parte de la información sobre cambio climático en el entorno online se publica en los días laborales $(84,8 \%)$ en comparación a los fines de semana $(15,2 \%)$. En el Grafico 1 puede verse que los picos se corresponden con los días laborables y los valle con los fines de semana. Este hecho nos que nos indica que existe una tendencia en la comunicación del cambio climático en el entorno online similar a la comunicación mediática convencional en cuanto al incremento del volumen de publicaciones en los días laborables.

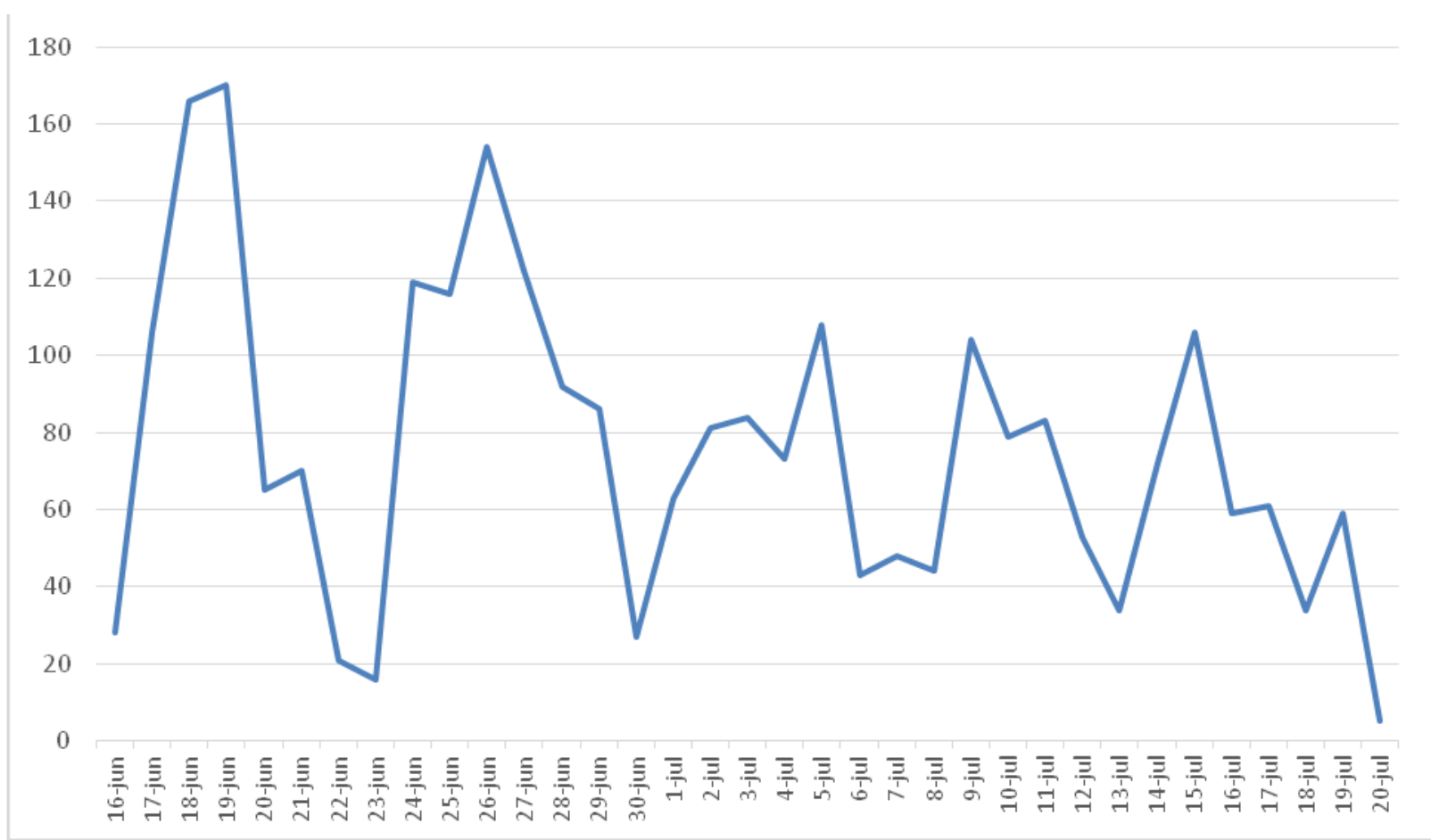

Gráfico 1. Comunicación online sobre cambio climático en España entre el 16/06/2019 y el 20/07/2019

Fuente: elaboración propia

\subsection{Tipología de las fuentes de información en Internet sobre el cambio climático}

Se han identificado 721 fuentes distintas que dieron lugar a las 2651 publicaciones. Con el 61,2\% de las publicaciones, la prensa online se sitúa como la principal fuente de información sobre cambio climático en España en Internet. Esta categoría no solo comprende a las versiones digitales de importantes diarios convencionales de referencia en soporte de papel, sino que también incluye a diarios nativos digitales, interesados en la cobertura de ámbito nacional, regional o local dependiendo del área geográfica de la audiencia a la que se dirigen (véase el Gráfico 2). Las "instituciones $u$ organizaciones que no son medios de comunicación" son la siguiente fuente de información más abundante al publicar el 19,3\% de las informaciones sobre cambio climático en el entorno online. Con un 5,3\% del total de las publicaciones encontradas, las agencias de noticias son la tercera fuente de información sobre cambio climático en Internet, seguidas de las revistas de medios y de los sitios web de televisión y radio. En penúltimo lugar, encontramos las fuentes que hemos denominado "híbridos" al ser medios que no pueden clasificarse en una sola de las categorías anteriores. Finalmente, con tan solo un $2 \%$ tenemos la categoría NP que corresponde a aquellas fuentes cuya identidad ha sido imposible de determinar. 
Se han encontrado 512 informaciones, el 19,3\% del total, que son divulgadas por instituciones u organizaciones que no son medios de comunicación. El Gráfico 3 muestra los porcentajes correspondientes a las publicaciones que llevaron a cabo las distintas fuentes que se han incluido dentro de esta categoría. Encontramos que la mayor parte, el $53 \%$, corresponde a la comunicación efectuada por asociaciones que representan los intereses estratégicos de distintos sectores productivos. Por volumen de información, las siguientes organizaciones (no medios) que comunican en Internet son las empresas (15\%), seguidas en tercer lugar por la comunicación institucional emitida por los gobiernos locales, autonómicos o nacional. Los centros de investigación (6 \%), las fundaciones $(2 \%)$ y los sindicatos $(2 \%)$ registran menor volumen de publicaciones. Las publicaciones de las ONG sólo alcancen el $1 \%$ del total en este periodo.

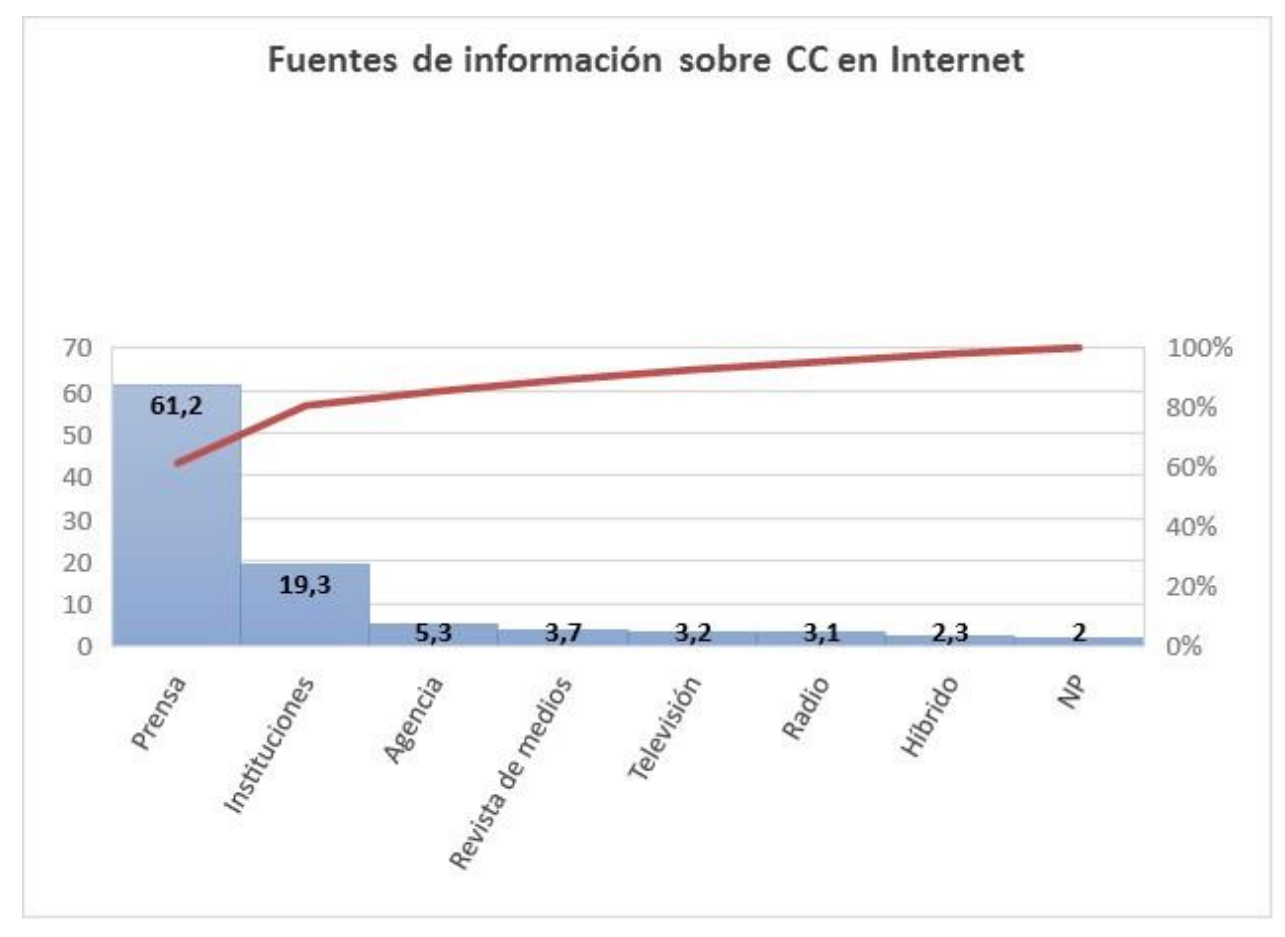

Gráfico 2. Porcentaje de publicaciones sobre cambio climático que corresponde a cada una de las distintas fuentes de información online

Fuente: elaboración propia 


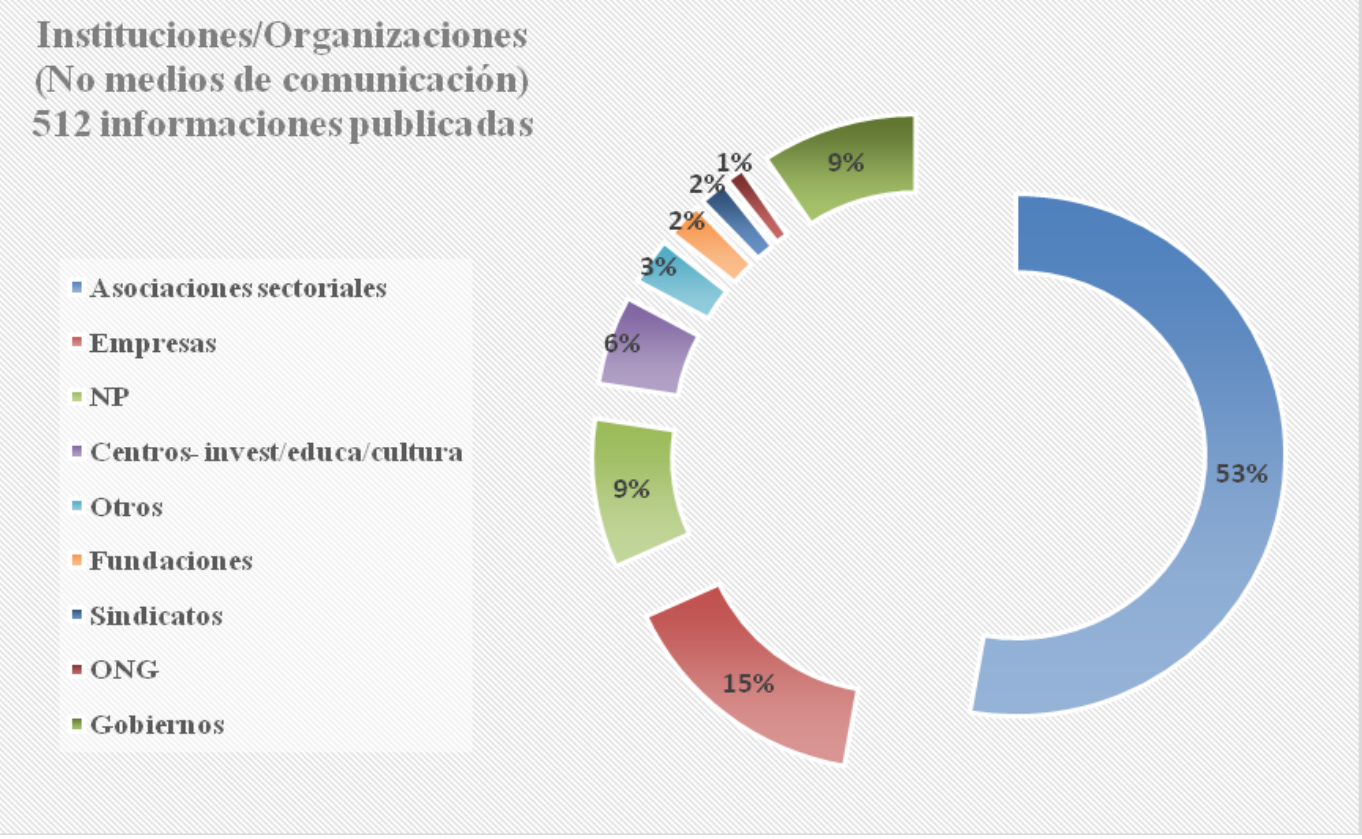

Gráfico 3. Porcentaje de publicaciones que corresponde a cada una de las distintas fuentes de información online sobre cambio climático que No son medios de comunicación

Fuente: elaboración propia

\subsection{Las primeras 30 fuentes de información sobre cambio climático en la comunicación online en España}

En el Gráfico 4 se ofrece el ranking de fuentes online que más informaciones publicaron sobre el cambio climático en España en la primera sonda del año 2019. En primer lugar, encontramos la agencia de noticias Europa Press con 80 publicaciones, un $3 \%$ del total, y en último lugar a aquellas fuentes con 15 publicaciones, un 0,6 \% del total, como es el caso de @ambientum.com, @elpais y @ cadenaser.com. A Europa Press le sigue la versión digital del periódico La Vanguardia en segunda posición con 53 publicaciones y un $2 \%$ del total de los registros encontrados. A continuación, se colocan varios diarios online como: Gente Digital, ABC, Eldiario.es, 20 Minutos y Diario Vasco. La $C O P E$ es la radio que lidera este soporte convencional en este ranking de comunicación online. Por su parte, Yahoo es news, Menéame y MSN España son los principales portales de información que forman parte de esta lista. El Economista, Expansión, Econoticias, Iagua.es y Ambientum son los representantes de la prensa especializada y Bolsamanía junto a @CCMA.CAT son las representantes instituciones que aparece en este ranking a pesar de no ser medios de comunicación. 


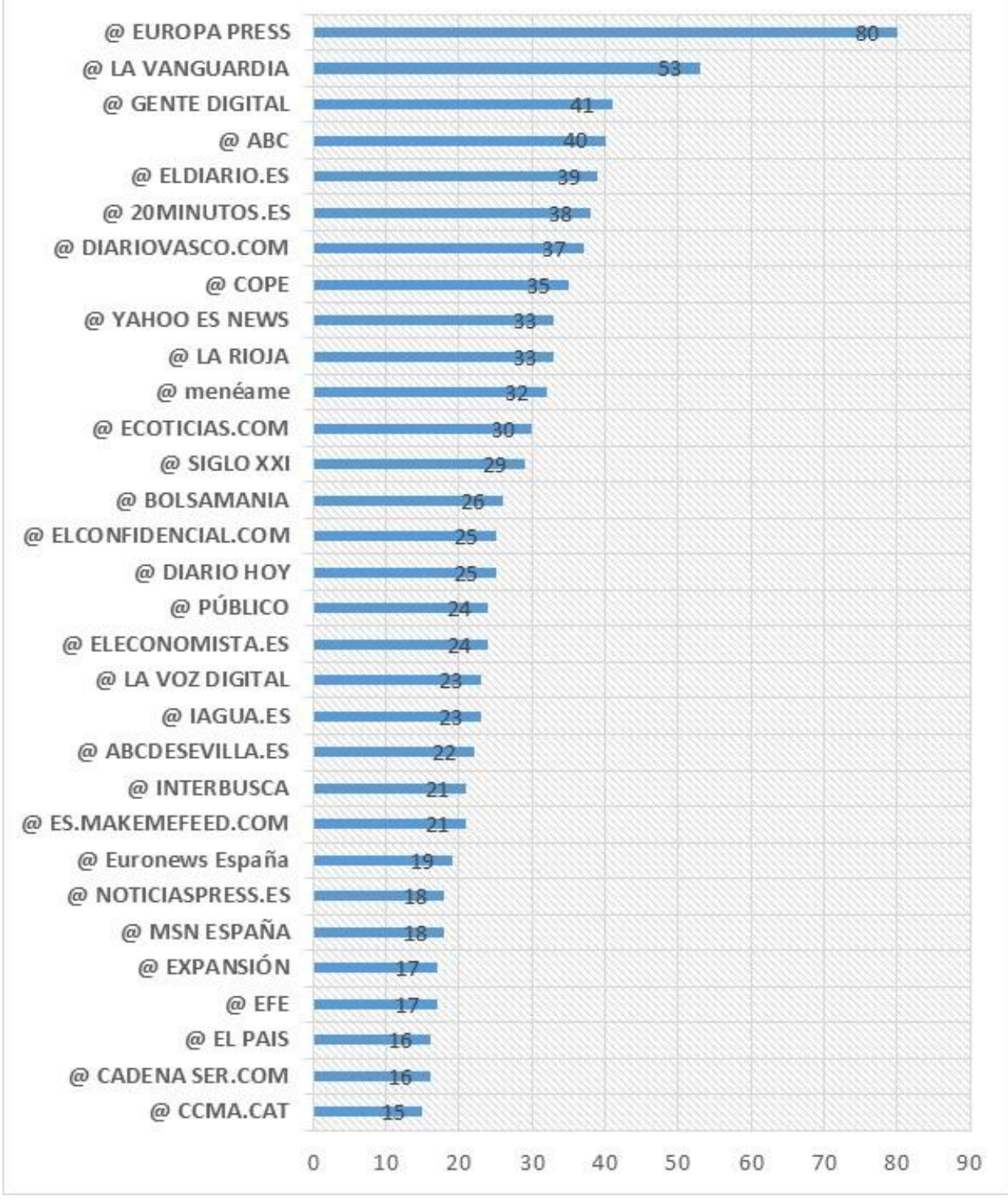

Gráfico 4. Ranking de fuentes online que más informaciones publicaron sobre cambio climático Fuente: elaboración propia

\subsection{Cobertura territorial de interés}

La comunicación online en España ofrece información de carácter local, regional o nacional que permite identificar el interés del medio o de la institución que comunica a la hora de dirigir su información a la audiencia de un determinado territorio, si bien, cualquier información publicada en la red de redes es accesible para cualquier ciudadano en cualquier territorio del planeta. En este sentido, la información online publicada sobre el cambio climático mantiene casi una simetría cuantitativa entre la cobertura nacional $(48,8 \%)$ y la cobertura regional-local $(41 \%)$, mientras que la cobertura internacional $(8,2 \%)$ queda muy atrás. En el Gráfico 5 puede observarse que la implicación territorial de los medios sigue estando muy presente en la comunicación online del cambio climático. 


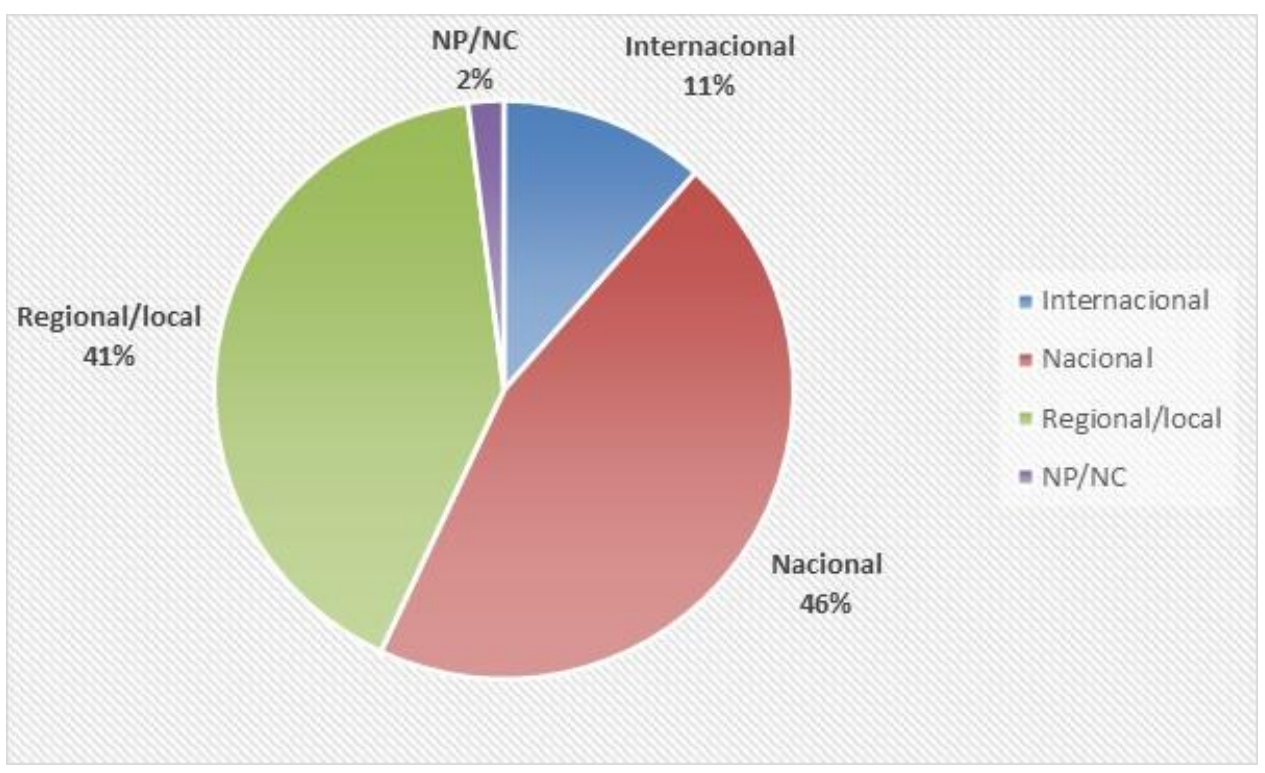

Gráfico 5. Porcentaje de fuentes que publican información de carácter local, regional o nacional Fuente: elaboración propia

La abundancia de fuentes de información online centradas en contenidos de carácter regional o local demuestra que trabajan con el objetivo de satisfacer a unos usuarios que presumen interesados por la información de proximidad y que reflejan la identidad social y geográfica de territorios concretos.

\subsection{Las fuentes de información online generalistas vs especializadas y cobertura de interés}

De las 2651 informaciones recogidas en los 35 días de la sonda analizada, en 52 casos no se ha podido identificar el tipo de fuente, 1936 registros corresponden a informaciones publicadas por fuentes de carácter generalista y 663 publicaciones fueron realizadas por fuentes especializadas en contenidos específicos, si bien se han encontrado algunas fuentes especializadas en audiencias de una determinada franja de edad (niños o mayores de 65 años).

En base a lo anterior, podemos decir que tres de cada cuatro referencias al cambio climático en la comunicación online en España se han realizado desde fuentes generalistas y una cuarta parte se realiza desde fuentes con discursos especializados en un determinado contenido. En el caso de la prensa, la fuente de información sobre cambio climático más importante en Internet, encontramos que nueve de cada diez referencias se hacen desde medios generalistas. Por el contrario, las instituciones $\mathrm{u}$ organizaciones que no son medios de comunicación ofrecen mayormente una información altamente especializada en un determinado sector o tema (véase Tabla 2).

Respecto al carácter local, nacional o internacional de la información publicada por las distintas fuentes, encontramos que las fuentes con interés en la cobertura nacional son la prensa generalista y las instituciones u organizaciones que no son medios de comunicación (asociaciones sectoriales y empresas fundamentalmente) y que se encuentran especializadas en un determinado sector productivo o contenido temático. Los medios con interés en la cobertura asuntos de carácter regional o local publican mayoritariamente información generalista y se trata sobre todo de prensa online. Son minoritarias las fuentes online interesadas en la cobertura de temas internacionales. 


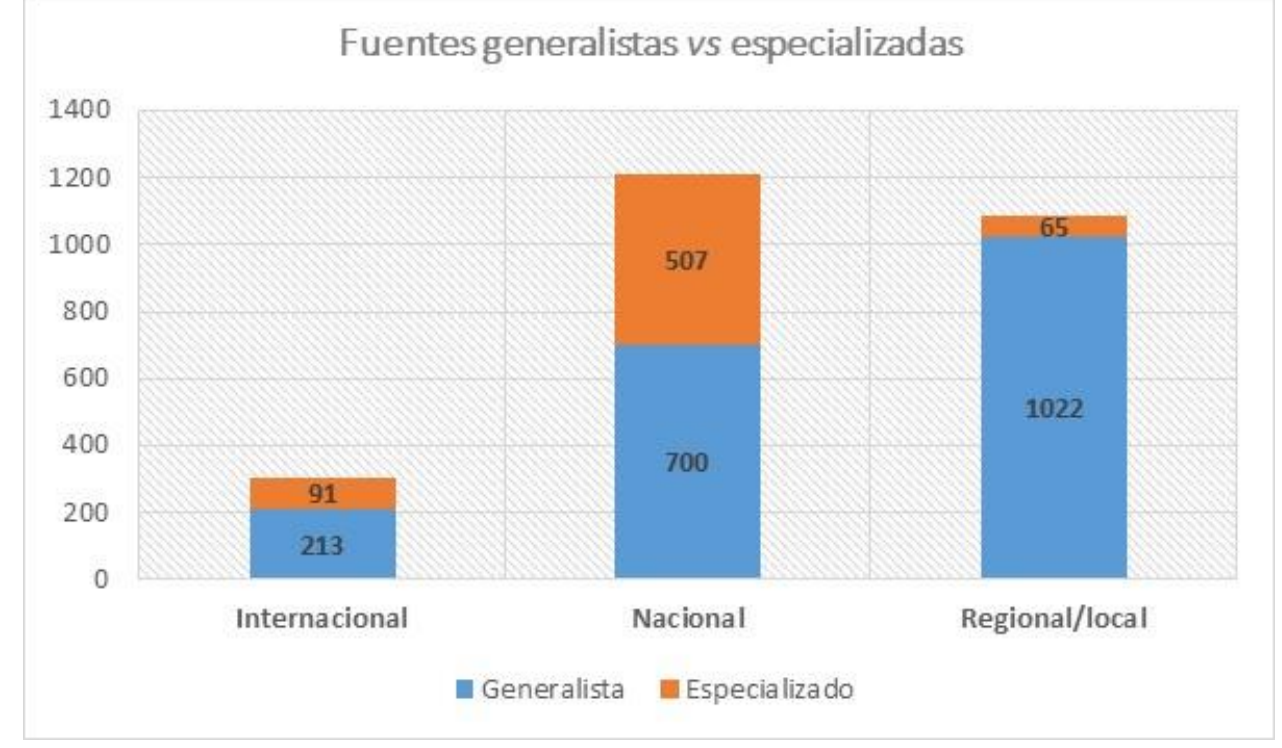

Gráfico 6. Publicaciones de carácter local, nacional o internacional realizadas por medios generalistas versus especializados

Fuente: elaboración propia

\subsection{Las fuentes de información online especializadas y la información del cambio climático}

Los datos expuestos en el apartado anterior nos indican que las publicaciones sobre cambio climático que provienen de fuentes especializadas constituyen el $25 \%$ del total. En la Tabla 2 se ofrecen los datos sobre los contenidos especializados de las fuentes online que publicaron sobre cambio climático en junio y julio de 2019.

La prensa online especializada que más ha publicado sobre cambio climático ha sido la especializada en economía, seguida por los diarios centrados en agua o medio ambiente. En la tercera posición empatan los diarios especializados en los mercados financieros y en información cultural. Sin embargo, sucede exactamente lo contrario con el tipo de fuentes que denominamos instituciones u organizaciones que no son medios de comunicación. En este caso, nueve de cada diez menciones al cambio climático se hacen desde organizaciones o instituciones dedicadas a comunicar temas ambientales en primer lugar. En segundo lugar, se sitúan las fuentes especializadas en contenidos como la economía y a las finanzas, situándose en tercer lugar las fuentes especializadas en cultura y educación en tercer lugar como contenido. Otras fuentes especializadas que comunicaron el cambio climático se encuentran orientadas a la cobertura de temas tan diversos como la defensa, los deportes, la educación, la energía, la informática, moda y estilo, política, religión, RSE, salud, seguridad, tecnología, trabajo y turismo. 
RLCS, Revista Latina de Comunicación Social, 80, 65-87

[Investigación] DOI: 10.4185/RLCS-2022-1531| ISSN 1138-5820| Año 2022

Tabla 2. Fuentes online de contenido especializado que publicaron sobre cambio climático

\begin{tabular}{|l|c|c|c|c|c|c|c|c|}
\hline $\begin{array}{c}\text { Contenido } \\
\text { especializado }\end{array}$ & $\begin{array}{c}\text { Medios } \\
\text { Híbrido }\end{array}$ & Prensa & Radio & Televisión & Agencia & $\begin{array}{c}\text { Revista } \\
\text { de } \\
\text { medios }\end{array}$ & $\begin{array}{c}\text { Instituciones } \\
\text { (no medios) }\end{array}$ & Total \\
\hline $\begin{array}{l}\text { Medio Ambiente/ } \\
\text { Agua }\end{array}$ & 0 & 29 & 0 & 0 & 7 & 10 & 118 & $\mathbf{1 6 4}$ \\
\hline Economía & 1 & 63 & 3 & 0 & 0 & 0 & 36 & $\mathbf{1 0 3}$ \\
\hline Cultura & 1 & 16 & 0 & 0 & 3 & 4 & 46 & $\mathbf{7 0}$ \\
\hline Sector primario & 1 & 8 & 1 & 0 & 1 & 0 & 51 & $\mathbf{6 2}$ \\
\hline $\begin{array}{l}\text { Mercado } \\
\text { financiero y Bolsa }\end{array}$ & 0 & 16 & 0 & 0 & 0 & 0 & 46 & $\mathbf{6 2}$ \\
\hline Educación & 0 & 1 & 0 & 0 & 0 & 0 & 41 & $\mathbf{4 2}$ \\
\hline Otros & 0 & 12 & 0 & 0 & 1 & 0 & 25 & $\mathbf{3 8}$ \\
\hline Energía & 0 & 4 & 0 & 0 & 0 & 0 & 21 & $\mathbf{2 5}$ \\
\hline Defensa & 0 & 1 & 0 & 0 & 0 & 0 & 18 & $\mathbf{1 9}$ \\
\hline Tecnología & 0 & 7 & 0 & 0 & 0 & 0 & 7 & $\mathbf{1 4}$ \\
\hline Moda y estilo & 5 & 5 & 0 & 0 & 0 & 0 & 0 & $\mathbf{1 0}$ \\
\hline Deportes & 0 & 4 & 0 & 0 & 0 & 0 & 4 & $\mathbf{8}$ \\
\hline Responsabilidad & 0 & 1 & 0 & 0 & 0 & 0 & 7 & $\mathbf{8}$ \\
\hline Religión & 0 & 0 & 0 & 0 & 5 & 0 & 1 & $\mathbf{6}$ \\
\hline Salud & 0 & 0 & 0 & 0 & 0 & 0 & 6 & $\mathbf{6}$ \\
\hline Turismo & 0 & 0 & 0 & 0 & 0 & 0 & 6 & $\mathbf{6}$ \\
\hline Prensa famosos & 0 & 5 & 0 & 0 & 0 & 0 & 0 & $\mathbf{5}$ \\
\hline Informática & 0 & 2 & 0 & 0 & 0 & 0 & 3 & $\mathbf{5}$ \\
\hline Seguridad & 0 & 2 & 0 & 0 & 0 & 0 & 3 & $\mathbf{5}$ \\
\hline Política & 0 & 0 & 0 & 0 & 0 & 0 & 3 & $\mathbf{3}$ \\
\hline Trabajo & 0 & 0 & 0 & 0 & 0 & 0 & 3 & $\mathbf{3}$ \\
\hline Total & $\mathbf{8}$ & $\mathbf{1 7 6}$ & $\mathbf{4}$ & $\mathbf{0}$ & $\mathbf{1 7}$ & $\mathbf{1 4}$ & $\mathbf{4 4 5}$ & $\mathbf{6 6 4}$ \\
\hline
\end{tabular}

Fuente: elaboración propia

En el Gráfico 7 puede apreciarse qué temas son referidos por las diversas fuentes online de información especializadas cuando comunican el cambio climático en España. Como puede apreciarse, son sobre todo las instituciones y las organizaciones especializadas en agua y medio ambiente las que más publicaciones realizaron sobre cambio climático. Sin embargo, la prensa especializada que más publicó sobre cambio climático fue la económica. 


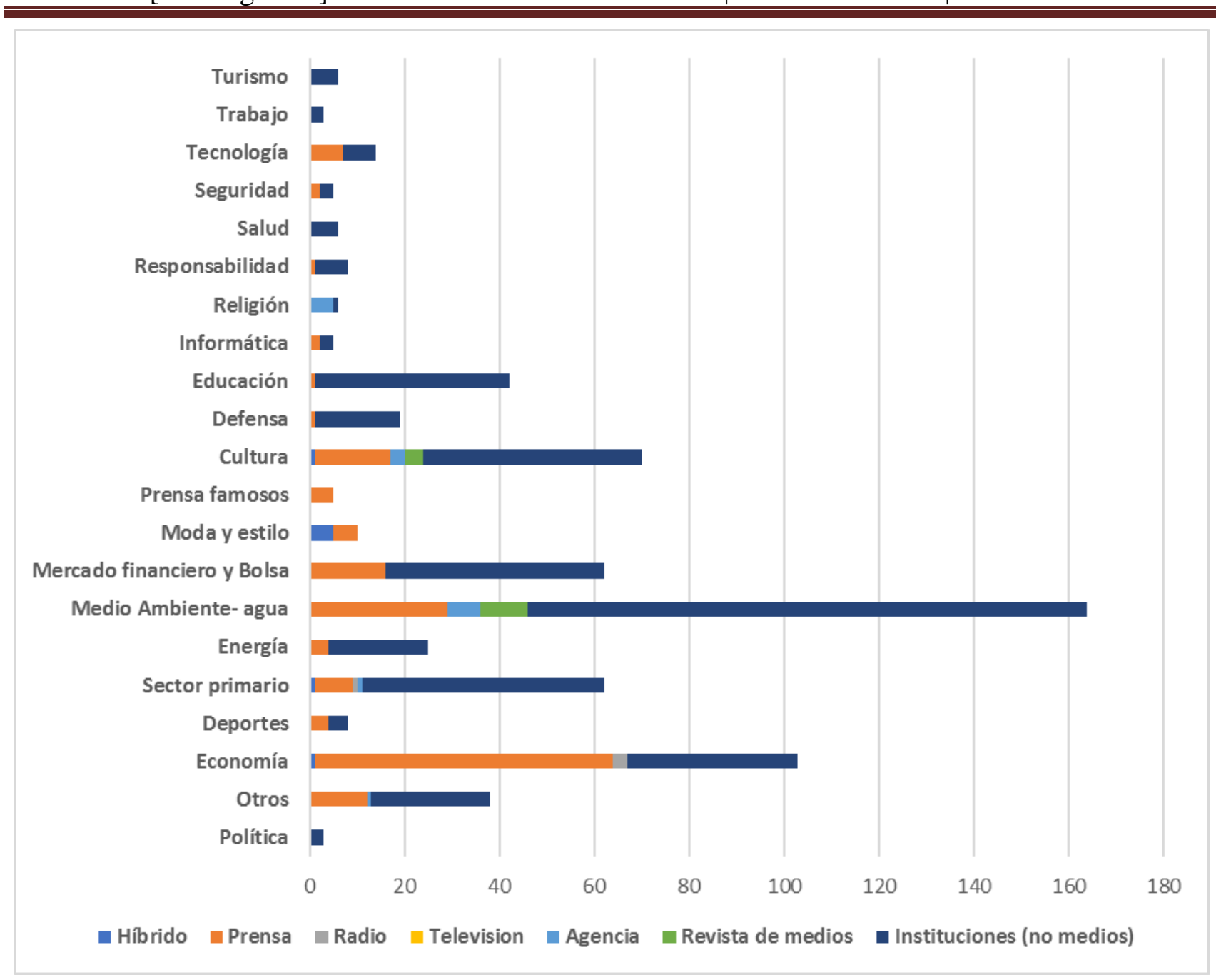

Gráfico 7. Volumen de publicaciones online sobre cambio climático de las fuentes distintas fuentes especializadas encontradas

Fuente: elaboración propia

\subsection{Respuesta a las preguntas de la investigación}

P.1. Teniendo en cuenta el volumen de medios online emergentes en la última década en España (Salaverria et ál., 2018), nos preguntábamos si también eran los medios de comunicación (radio, televisión, prensa) los principales emisores de información sobre el cambio climático en Internet. Los resultados confirman este hecho ya que sí son los medios de comunicación online las principales fuentes de información sobre el cambio climático en Internet, acumulando el $70 \%$ de las publicaciones sobre cambio climático identificadas. Ahora bien, no todos los soportes tienen la misma presencia en Internet. La prensa es líder indiscutible en cuanto al volumen de publicaciones $(61,1 \%)$, siendo muy inferior la frecuencia de la comunicación online sobre cambio climático de la televisión $(3,2 \%)$, la radio $(3,1 \%)$ y los medios híbridos $(2,3 \%)$. De estos dos últimos soportes, encontramos los sitios web de dos emisoras de radio que entran en el ranking de las 30 principales fuentes de información, pero ningún sitio web de televisión lo logra. Este último hecho contrasta con los estudios que señalan la relevancia que la población otorga a la televisión convencional como principal fuente de información sobre el cambio climático y que remarcan la gran influencia que mantiene la televisión convencional en la percepción social del cambio climático (Newman, Fletcher et ál., 2020). Las agencias de noticias publicaron el 5,3\% de las informaciones online encontradas. 
P.2. En segundo lugar, nos cuestionábamos sobre cuáles eran los agentes sociales e institucionales que se comportan como fuentes de información sobre cambio climático en el entorno online y sobre las características de su comunicación. Se han identificado 721 fuentes online distintas que han realizado un total 2561 publicaciones en el periodo analizado. Hemos encontrado que la prensa online es la responsable del $61,2 \%$ de las informaciones publicadas sobre cambio climático y que el $19,1 \%$ de estas informaciones son emitidas por asociaciones e instituciones que no son medios de comunicación, entre las que destacan las asociaciones sectoriales que defienden los intereses de sectores productivos concretos, los gobiernos regionales, autonómicos, el nacional y los centros de investigación en último lugar. En cuanto a la idiosincrasia de la entidad que comunica y su interés en la cobertura informativa de contenidos de un contexto territorial concreto, hemos encontrado que las fuentes interesadas en temas de cobertura nacional (48,8 \%) superan ligeramente a las fuentes de cobertura regional-local $(41 \%)$, mientras que la cobertura internacional es claramente minoritaria $(8,2 \%)$.

P.3. En tercer lugar, nos interesaba descubrir en qué temas están especializadas las diversas fuentes de información online que comunicaron sobre cambio climático en el periodo analizado. Los contenidos generalistas fueron los predominantes en las fuentes que más publican sobre cambio climático en Internet y que no es otra que la prensa online. Sin embargo, las asociaciones sectoriales y las empresas son las principales fuentes de información con contenidos especializados en temas y asuntos de muy diversa naturaleza (economía, deportes, sector primario, energía, medio ambiente, agua, finanzas, etc.).

\section{Discusión}

La comunicación online sobre el cambio climático en España está poco estudiada y se caracteriza por contar con una gran cantidad y diversidad de fuentes de información. Si bien es cierto que la quinta parte del total de esas informaciones son publicadas por instituciones que no son medios de comunicación, mientras que la mayor parte del total (el 70\%) sí son publicadas por medios profesionales, destacando abrumadoramente la prensa. Comprobamos que comunicar sobre el cambio climático es una práctica social que atañe de manera creciente tanto a los medios de comunicación como a las instituciones no mediáticas que también participan de estas prácticas. Por consiguiente, no siempre se realiza una cobertura informativa que responda a las rutinas y cánones periodísticos, sino que también se generan y difunden informaciones que responden a intereses sectoriales concretos que circulan entre nuevos públicos en paralelo a las audiencias mediáticas.

Entre las instituciones que no son medios de comunicación destacan las asociaciones sectoriales que defienden los intereses de sectores productivos concretos, los gobiernos regionales, autonómicos, el nacional y los centros de investigación en último lugar. Investigaciones anteriores han constatado que los representantes de las empresas han sido los grandes ausentes en medios como la televisión cuando se abordaba el cambio climático como referente informativo (Teso, Fernández-Reyes et ál., 2018; Teso, Gaitán et. ál., 2020). Esto apunta a que las asociaciones sectoriales y las empresas prefieren sus propios sitios web para comunicar en relación con el cambio climático, antes que intervenir para tomar la palabra en los medios de comunicación. En sentido inverso, nos encontramos con que las publicaciones de las ONG sólo alcanzan el $1 \%$ del total. Estas organizaciones son expertas en orientar sus estrategias de comunicación para captar la atención de los medios (Castillo, 2007) lo que podría explicar en cierto sentido esta baja actividad como fuente online, teniendo en consideración que este estudio no incluye las publicaciones en Redes Sociales, lugares en los que algunas ONG despliegan gran actividad comunicativa (García, Fernández \& Del Olmo, 2018). 
A pesar del elevado número de publicaciones analizadas (2561 unidades), los autores somos conscientes de la limitación temporal de este estudio correspondiente a la primera sonda de 2019. Tras este análisis exploratorio, nuestra intención es continuar con el estudio para proceder a la divulgación de los resultados de las sondas posteriores realizadas en los años 2019, 2020 y 2021. Los resultados de este primer estudio exploratorio nos permiten proponer nuevas hipótesis de trabajo.

Teniendo en cuenta el elevado volumen de publicaciones y fuentes encontradas, la primera hipótesis podría apuntar a un incremento del interés general y sectorial por el cambio climático que se tradujera en un incremento en el volumen de las publicaciones circulantes en el entorno online. Sin embargo, en primer lugar, debe analizarse cómo la pandemia causada por el SarsCov-2 ha impactado en la comunicación online del cambio climático, para confirmar si sigue la misma tendencia que en los medios convencionales en España (Fernández-Reyes \& Jiménez, 2020). En segundo lugar, a partir de los resultados obtenidos, podemos presumir que se irá incrementando progresivamente la cobertura informativa online del cambio climático de forma paralela, tanto por parte de los medios profesionales como a través de los canales empleados por sectores productivos y de servicios cuya actividad está relacionada con el cambio climático. Por otra parte, este incremento puede traducirse en la creación de nuevos contenidos y propuestas comunicativas dirigidas a nuevas audiencias que aporten el valor de la especialización para sectores concretos.

Este estudio nos ha permitido comprobar el peso del sector económico en la información especializada, por lo que será de gran interés comprobar si fenómenos vinculados con el cambio climático como son los eventos meteorológicos extremos son de interés para las fuentes especializadas en sectores estratégicos como la energía, el agua o el sector financiero. Por último, de acuerdo con el trabajo realizado por Boehnert (2014) y Almirón, Boykoff, et ál. (2020), sería de gran interés analizar específicamente en qué medida tienen presencia fuentes negacionistas en el entorno online y a qué grupos de interés responden.

\section{Conclusiones}

Los medios de comunicación online constituyen las principales fuentes de información sobre el cambio climático en Internet (excluyendo las redes sociales). Las fuentes que más referencias al cambio climático realizan son la prensa generalista, seguida de la prensa especializada, las instituciones que no son medios de comunicación (asociaciones sectoriales, empresas e instituciones oficiales fundamentalmente), las agencias de noticias, los portales de medios, y los sitios de web de radio y televisión. La histórica agencia de noticias Europa Press ocupa el primer lugar por volumen de información publicada en el ranking de las 30 principales fuentes de información online sobre cambio climático. Es significativo el escaso volumen de publicaciones online de la televisión y de las ONG.

Mientras que en la prensa online predominaron los contenidos generalistas, las publicaciones especializadas corresponden fundamentalmente a instituciones u organizaciones que no son medios de comunicación. Las fuentes especializadas que publicaron contenidos sobre el cambio climático constituyen una cuarta parte del total e incluyen tanto a los medios profesionales como a otras fuentes. Si sumamos el volumen de publicaciones que acumulan las fuentes especializadas en economía y finanzas con las del sector primario encontramos que más de un tercio de las publicaciones sobre cambio climático relacionaban este fenómeno con la actividad económica. Las fuentes dedicadas a la publicación de contenidos educativos y culturales se ocuparon también del cambio climático. Encontramos una gran variedad de sectores especializados concernidos por la transformación ecológica anunciada en un contexto social de una emergencia climática que fue declarada oficialmente por el gobierno español en enero de 2020. 
El medio ambiente y la economía (incluyendo las finanzas) tienen un gran peso en el volumen de publicaciones especializadas. Esto constituye un indicador de la relevancia alcanzada por el cambio climático como asunto de interés público en el año 2019, que aparece informaciones publicadas por fuentes especializadas en sectores y temas tan dispares como la religión, la moda, la defensa o el turismo. Además de esta relevancia sectorial, este hecho pone de manifiesto la transversalidad del cambio climático como problemática ambiental, social y económica, así como la necesidad de planes integrales y sectoriales para combatir sus impactos y mejorar la adaptación en los distintos sectores.

En relación con las características de las fuentes de información sobre cambio climático en el entorno online, encontramos que los cibermedios ofrecen un contenido de marcado carácter generalista mientras que las instituciones que no son medios publican en mayor medida contenidos especializados en gran diversidad de temas y sectores. En cuanto al interés por divulgar contenidos ligados a contextos territoriales concretos, encontramos un equilibrio cuantitativo entre las fuentes que publican contenidos de ámbito nacional y aquellas centradas en contenidos territoriales. Encontramos un elevado volumen de fuentes que publican desde distintas Comunidades Autónomas con lengua propia cuyos sus contenidos están centrados en la cobertura de temas de interés para dichos territorios.

Por último, cabe destacar que se mantienen ciertos rasgos estructurales que también condicionan la comunicación del cambio climático en los medios convencionales, como son la influencia de la estacionalidad y el descenso del volumen de información los fines de semana, fenómeno ya señalado por Arcila et al. (2015). Siendo la prensa online la principal fuente de información sobre el cambio climático en Internet, resulta evidente esta tendencia que reproduce los patrones de comportamiento de los medios convencionales en el contexto online.

\section{Referencias}

Almirón, N.; Boykoff, M.; Naberhous, M. \& Heras, F. (2020). Dominant counter-frames in influential climate contrarian European think tanks. Climatic Change, 162. https://doi.org/10.1007/s10584-020-02820-4

Arcila, C.; Mercado, M.T.; Piñuel, J. L. \& Suarez-Sucre, E. (20015). Media coverage of climate change in spanish-speaking online media. Convergencia. Revista de Ciencias Sociales, 22 (68), 71-95.

Boehnert, J. (2014). Mapping Climate Communication $N^{o}$ 2, Network of Actors: USA, UK and Canadian Based Institutions, Organizations and Individuals. Center for Science and Technology Policy Research, Cooperative Institute for Research in Environmental Sciences (CIRES). University of Colorado Boulder. http://ecolabsblog.wordpress.com

Castillo, A. (2007). Relaciones Públicas en las organizaciones no gubernamentales. SPHERA PUBLICA. Revista de Ciencias Sociales $y$ de la Comunicación, 7. https://www.redalyc.org/pdf/297/29720421012.pdf

Díaz, B. (2011). Libro negro del periodismo en España. Madrid: Asociación de la Prensa de Madrid.

Díaz-Noci, J., Salaverría, R. (coords.) (2003). Manual de redacción ciberperiodística. Barcelona: Ariel. 
Fernández-Reyes, R. (2019). Crónica de la cobertura del cambio climático en prensa de 2019. https://recambiacambioclimatico.files.wordpress.com/2020/01/16.-resumen-2019.pdf

Fernández-Reyes, R. \& Teso, G. (2019). Resumen de diciembre 2019 de la cobertura mediática del cambio climático. https://recambiacambioclimatico.com/2020/01/04/resumen-diciembre-2019/

Fernández-Reyes, R. \& Jiménez, I. (2020). Spanish Newspaper Coverage of Climate Change or Global Warming, 2000-2020. Cooperative Institute for Research in Environmental Sciences.

https://doi.org/10.25810/37f9-1j65.30

Francescutti, L.P., Tucho, F. \& Íñigo, A. I., (2013). El medio ambiente en la televisión española: análisis de un año de informativos. Estudios sobre el Mensaje Periodístico, 19 (2), 683-701. Servicio de Publicaciones de la Universidad Complutense, Madrid.

Gaitán, J.A. \& Piñuel, J. L. (2013). Efectos de la crisis en el discurso sobre el Cambio Climático desde Cancún a Durban. Revista Disertaciones, 6, (1), 172-189.

García, C., Fernández, C. \& Del Olmo, J. (2018). La comunicación del Tercer Sector y el compromiso de los jóvenes en la era digital. ZER - Revista de Estudios de Comunicación, Vol. 23 (44). https://ojs.ehu.eus/index.php/Zer/article/view/19164/17678

Heras, F., Meira, P.A. \& Benayas, J. (2016) Un silencio ensordecedor. El declive del cambio climático como tema comunicativo en España 2008-2012. Redes.com: revista de estudios para el desarrollo social de la Comunicación, 13, pp. 31-56.

IPCC (2019). Special Report on the Ocean and Cryosphere in a Changing Climate. https://www.ipcc.ch/srocc/home/

León, B. \& Erviti, M. C. (2015). Science in pictures: Visual representation of climate change in Spain's television news. Public Understanding of Science, Vol. 24 (2), 183- 199.

Lopera, E. (2013). La comunicación social de la ciencia del clima en la prensa española: texto y contexto. Tesis doctoral. Universidad de Valencia, Valencia.

López, G. (2015). Periodismo digital. Redes, audiencias y modelos de negocio. Salamanca: Comunicación Social.

Lozano, C. (2013). El cambio climático en los telediarios: alusiones a la catástrofe en tiempos de calma. Disertaciones, Vol. 6 (1), 124-140. https://bit.ly/3mfnsYG

Mancinas, R. (2013). El silencio mediático. Reflexión en torno a las razones de los medios de comunicación para no hablar del cambio climático, en Fernández, R. (Director), Mancinas, R. (Coordinadora), Medios de Comunicación y cambio climático, Sevilla: Fenix Editora, 233-248.

Meso, K. (2006). Introducción al ciberperiodismo. Breve acercamiento al estudio del Periodismo en Internet. Bilbao. Servicio de Publicaciones de la Universidad del País Vasco. 84-8373-861-9 
Newman, N., Fletcher, R., Schulz, A., And1, S. \& Kleis Nielsen, R. (2020). Digital News Report 2020. Reuters Institute for the Study of Journalism. University of Oxford. https://reutersinstitute.politics.ox.ac.uk/sites/default/files/2020-06/DNR_2020_FINAL.pdf

Parratt, S. (2009). Climate change in Spain's media: A deficient answer. Infoamérica-Iberoamerican Communication Review, 1, 129-138.

Rejón, R. (2018). Bolsonaro se une a Donald Trump en el eje que mina la lucha contra el cambio climático. Eldiario.es https://www.eldiario.es/sociedad/bolsonaro-trump-lucha-cambioclimatico_1_1886629.html

Teso, G., Fernández- Reyes, R., Gaitán, J. A., Lozano, C. \& Piñuel, J. L. (2018). Comunicación para la sostenibilidad, el cambio climático en los medios. Documento de Trabajo Sostenibilidad $\mathrm{n}^{\circ}$ 1/2018. Madrid. Fundación Alternativas. https://bit.ly/34rSAOs

Teso. G., Gaitán, J. A., Lozano. C., Fernández-Reyes, R., Sánchez-Holgado, P., Arcila, C., Morales E. \& Piñuel, J.R. (2020). Diseño del observatorio de la comunicación mediática del cambio climático. Madrid, Fundación ECODES. Documento en la red: https://ecodes.org/images/quehacemos/pdf_MITECO_2019/INFORME_OBSERVATORIO_COMUNICACION_CC.pdf

Salaverría, R., Martínez-Costa, M.P. \& Breiner, J. (2018). Mapa de los cibermedios de España en 2018: análisis cuantitativo. Revista Latina de Comunicación Social, 73, 1034-1053. 10.4185/RLCS-2018-1295

Salaverría, R. (2020). Digital journalism: 25 years of research. Review article. El profesional de la información, V. 28, n. 1, e280101. https://doi.org/10.3145/epi.2019.ene.01

Yunquera, J. (2016). Revistas y diarios digitales en España: historia de una evolución. Barcelona: Editorial UOC.

\section{AUTORES:}

\section{Gemma Teso Alonso}

Doctora en Sociología por la Universidad Complutense de Madrid. Licenciada en Comunicación Audiovisual y Experta en elaboración de Reportajes y Documentales (UCM). Profesora de Teoría de la Comunicación y Metodología de Investigación en Comunicación Social en la Facultad de Ciencias de la Información (UCM). Funcionaria docente de carrera de la especialidad de Procesos y Medios de Comunicación de la Consejería de Educación de la CAM. Trabajó 10 años en el ente público Radio Televisión Madrid. Coordinadora del Observatorio de la Comunicación Mediática del Cambio Climático puesto en marcha por el grupo de investigación Mediación Dialéctica de la Comunicación Social (MDCS) de la UCM y ECODES. Ha sido investigadora del I+D+i "El discurso hegemónico de los medios sobre el cambio climático (Riesgo, incertidumbre y conflicto) y prueba experimental con discursos alternativos entre jóvenes (CSO2010-16936COMU)". Entre otros, ha coordinado el proyecto de innovación aplicada del $\mathrm{M}^{\mathrm{o}}$ de Educación "Jóvenes frente al Cambio Climático" (www.jovenesfrentealcambioclimatico.com) y la Asociación Estratégica K202 Erasmus + para la Innovación educativa y el intercambio de buenas prácticas YOUNGSTERS I.DOC MAKERS (YIDOCM, https://goo.gl/wv6CbE). Miembro del equipo de investigación del proyecto MAPCOM 2. gtesoalonso@gamil.com

Índice h: 8 
Orcid ID: https://orcid.org/0000-0001-9852-0255

Google Scholar: https://scholar.google.com/citations?user=yZ8yRhQAAAAJ\&hl=es\&oi=ao

\section{Carlos Lozano Ascencio}

Doctor en Ciencias de la Información por la Universidad Complutense de Madrid y Licenciado en Ciencias de la Comunicación por la Universidad Nacional Autónoma de México. Es profesor Titular de Universidad de Periodismo en la Universidad Rey Juan Carlos (URJC). En la actualidad dirige el Máster Universitario en Periodismo Cultural y Nuevas Tendencias de la URJC y es Coordinador de la Sección: Teorías y métodos de investigación en Comunicación de la Asociación Española de Investigación en Comunicación (AE-IC). Sus principales líneas de investigación tienen que ver con la Comunicación del Riesgo y de la Catástrofes, la Meta-investigación de la Comunicación y la Historia del Periodismo. Miembro del equipo investigador del Observatorio de la Comunicación Mediática del Cambio Climático puesto en marcha por el grupo de investigación Mediación Dialéctica de la Comunicación Social (MDCS) de la UCM y ECODES. Es Investigador Principal (IP) del proyecto MAPCOM 2 (https://2.mapcom.es/).

carlozanoas@gmail.com

Índice h: 15

Orcid ID: https://orcid.org/0000-0003-2537-0799

Google Scholar: https://scholar.google.com/citations?user=ioALM-AAAAAJ\&hl=es\&oi=ao 